\title{
Competência informacional e medicina baseada em evidências
}

\author{
Information literacy and evidence-based medicine
}

\author{
Rosana EVANG ELISTA ${ }^{1}$ \\ Vanda de Fátima Fulgêncio de O LIVEIRA ${ }^{2}$ \\ Sandra Lúcia PEREIRA ${ }^{3}$ \\ Valdinéa Sonia PETINARI ${ }^{4}$
}

RESUMO

As pesquisas acadêmicas e aquelas envolvendo a tomada de decisões clínicas a presentam-se de forma justaposta na prática dos pesquisado res da área da saúde, necessitando a perfeiçoamento e especialização de novas habilidades essenciais aos profissionais da informação. Partindo dessa premissa, resolvemos investigar, na literatura da área, publicações que tratassem ao mesmo tempo das temáticas: Medicina Baseada em Evidências e Information Literacy/C ompetência Informacional. 0 objetivo deste trabalho foi não apenas conceituar Medicina Baseada em Evidências e Information Literacy/C ompetência em Informação por meio de revisão de literatura, como também assinalar a confluência dessas temáticas na prática dos pesquisadores da área da saúde. Como resultado desta análise, verificou-se que o profissional da informação necessita conhecer seus usuários, o modo como a informação se organiza e como ela se apresenta, para obter melhores resultados de busca, reduzir incertezas e, ao mesmo tempo, proporcionar aos pesquisadores da área da saúde maior suporte para tomada de decisão. Concluiu-se que uma demanda por informação exige, diretamente, que o profissional da informação aplique sua competência para atendê-la, recorrendo a sua capacitação e conhecimento dos meios para chegar à info rmação adequada, utilizando as ferramentas corretas, respondendo à demanda sem deixar dúvidas de confiabilidade. Esse profissional, como mediador, muito contribui para a prática da Medicina Baseada em Evidências e para a divulgação de serviços e acessos, educação e promoção da competência em informação dos pesquisadores.

Palavras-chave: competência informacional; medicina baseada em evidências; profissional da informação.

\footnotetext{
Mestre em Biblioteconomia, Pontifícia Universidade Católica de Campinas. Bibliotecária, Faculdade de Ciências Médicas, Universidade Estadual de Campinas. Rua Tessália Vieira Camargo, 126, Cidade Universitária "Prof. Zeferino Vaz", Barão G eraldo, 13084 971, Campinas, SP, Brasil. Correspondência para/Correspondende to: R. EVANGELISTA. E-mail: < rosanae@ fcm.unicamp.br>.

2 Graduada em Ciências Sociais e Biblioteconomia, Pontifícia Universidade Católica de Campinas. Bibliotecária, Centro de Atenção Integral à Saúde da Mulher (CAISM), Universidade Estadual de Campinas. Campinas, SP, Brasil. E-mail: < vanda@ unicamp.br> .

3 Mestre em Biblioteconomia - PUC-Campinas. Bibliotecária da Faculdade de Ciências Médicas da UNICAMP. $<$ sandralu@fcm.unicamp.br>.

4 Graduada em Tecnologia em Processamento de Dados, UNISAL. Especialista em G erência de Sistemas e Serviços de Informação. Graduanda em Ciência da Informação com Habilitação em Biblioteconomia, Pontifícia Universidade Católica de Campinas. Responsável pela Central de Referência em Equipamentos Biomédicos do CEB, Universidade Estadual de Campinas. Campinas, SP, Brasil.<val@ ceb.unicamp.br>. Recebido em 3/1/2007 e aceito para publicação em 1/8/2007.
} 


\begin{abstract}
The academic research and those involving the clinical decisions making are presented on juxtaposed form in health-care providers' practice, demand improvements and specialization of new necessary abilities to the professionals of information. Starting from this premise, we decide to investigate in the literature the publications in the areas that dealt, at the same time, with the thematic ones: Evidence-Based Medicine and Information Literacy. The objective of this work was to concept Evidence-Based Medicine and Information Literacy through literature revision and to designate the confluence of these thematic ones in health care providers' practice. As a result of this analysis, it was verified that the professional of information needs to know the users, how the information is organized and how it presents itself, to get better search results, to reduce uncertainties and at the same time to provide to these researchers a bigger support for decision making. It was concluded that a demand for information needs directly that the professional of information applies the ability to take care appealing it to both qualification and knowledge to get to the suitable information, using the correct tools, answering the demand without leaving trustworthiness doubts. This professional, as a mediator, contributes very much for the practice of the Evidence-Based Medicine and the spreading of services and accesses to it as well as education and promotion.
\end{abstract}

Keywords: information literacy; evidence-based medicine; professional of information.

\section{INTRO DUÇÃO}

A evolução da ciência e da tecnologia afeta diretamente a vida cotidiana, e as profissões não estão a salvo da mudança imposta. Hoje as organizações estão sendo desafiadas a lidar com ambientes cada vez mais dinâmicos, demandando novas exigências de gestão e novos perfis de lideranças. G rande ênfase tem-se dado à questão das competências essenciais da organização, que se referem ao aprendizado coletivo, e das competências do indivíduo.

Sabemos que o bibliotecário atual busca qualificação e um aprendizado permanente, visto que "cada vez mais este profissional é exigido por seus usuários, e para tanto necessita estar apto a transmitir, de forma viável, informações relativas à busca de informação" (Passos; Santos, 2005, p.18). Neste trabalho usaremos as denominações bibliotecário e profissional da informação como sinônimas.

Especialistas da informação no Brasil apontam as competências requeridas aos profissionais da informação para essa nova configuração, das quais destacamos aqui: conhecimento interdisciplinar e especializado; capacidade de contextualização; capacidade de conceituação; conhecimento da demanda ou do cliente; domínio de tecnologias de informação; adaptação ao novo; flexibilidade e abertura às mudanças (Faria et al., 2005).
Para avaliar seus conhecimentos, habilidades e atitudes os profissionais da informação estão utilizando instrumentos como o Mapeamento de Competência, buscando indicadores que auxiliem a aprendizagem da categoria sobre si mesma e possibilite aos profissionais autogerenciarem suas carreiras (Faria et al., 2005; O liveira et al., 2006).

Para que o profissional da informação desenvolva as competências necessárias às exigências atuais no trato e disseminação da informação, é imprescindível o conhecimento das necessidades informacionais dos indivíduos ou grupos que fazem parte da comunidade usuária de uma determinada unidade de informação (Passos; Santos, 2005).

Uma demanda por informação exige, diretamente, que o profissional da informação aplique sua competência para atender essa demanda recorrendo a sua capacitação e conhecimento dos meios para chegar à informação adequada. Para tanto, utilizar as ferramentas corretas faz parte do julgamento do profissional que responderá à demanda sem deixar dúvidas de confiabilidade.

Conforme Le Coadic (2004, p.138), utilizar um produto de informação é obter um efeito que satisfaça a uma necessidade de informação.

0 objetivo final de um produto de informação ou de um sistema de informação deve ser pensado em termos dos usos dados à informação 
e dos efeitos resultantes desses usos nas atividades dos usuários. A função mais importante do produto ou do sistema é, portanto, a forma como a informação modifica a realização dessas atividades. Por causa disso, devem ser "orientadas para o usuári".

Existe uma associação entre o modelo de pesquisa utilizado para responder cada pergunta e 0 nível ou validade da evidência obtida. Para Le Coadic (2004, p.43), é preciso que conheçamos as circunstâncias que levam o usuário a iniciar um processo de busca de informações, se quisermos compreender os fenômenos que ocorrerão quando do uso dos distintos sistemas, serviços e produtos mobilizados pelo usuário. A consulta que o usuário formulará ao sistema, a interação que ocorrerá entre o usuário e o sistema (ou o intermediário) e a avaliação do êxito ou prejuízo dessa interação dependerão dessas circunstâncias.

Na prática, a análise das necessidades é uma atividade interativa que alterna coleta de dados (coleta direta e/ ou indireta por meio de medições e levantamentos), análise desses dados e decisão.

\section{O Bibliotecário em Ciências da Saúde}

As pesquisas para fins acadêmicos e as pesquisas para tomada de decisões clínicas a presentamse de forma justaposta na prática dos pesquisadores da área da saúde, necessitando do aperfeiçoamento e especialização das habilidades dos profissionais da informação que atuam na área da saúde.

Podemos considerar que a informação demandada para a pesquisa em saúde e para a tomada de decisão clínica deve ser metodologicamente estruturada, apresentando-se como informação científica. Existe também a demanda de informação para a gestão em saúde, não sendo necessariamente científica, mas também fundamental para o processo decisório inerente à gestão em saúde (Pinto, 2005).

A atuação do bibliotecário na área da saúde expande-se na medida em que são utilizadas novas ferramentas de pesquisa para dar suporte aos profissionais da área da saúde, sendo a atualização uma das competências profissionais mais exigidas na área. Percebe-se que esses profissionais têm uma tendência a assumir cada vez mais responsabilidades junto ao processo de tomada de decisão (Bueno; Blattmann, 2005; Pinto, 2005; Silva, 2005).

0 médico, de acordo com Atallah (2001a), ao tomar uma decisão em relação ao problema de saúde de um paciente, um grupo de pacientes, uma comunidade ou um país, sabe que precisa embasar-se na melhor evidência científica conhecida.

No início da década de 1990 com o surgimento da Medicina Baseada em Evidências - MBE -, o papel do bibliotecário ganha destaque na equipe médica. Todo o processo de busca, seleção e avaliação crítica da literatura, para responder a questões clínicas que permeiam o conceito de $\mathrm{MBE}$, favorece a integração do bibliotecário junto à equipe médica, sendo atualmente assunto de vários artigos científicos quanto às competências para sua formação, às funções que desempenha nos processos de uma revisão sistemática, à efetividade da sua atuação no meio clínico, entre outras abordagens (Pinto, 2005).

Considerando esse contexto, fomos motivadas para esta análise que envolve duas grandes temáticas no contexto informacional atual: de um lado, a Medicina Baseada em Evidências e, de outro, a Competência em Informação.

Essas temáticas apresentam origens e características diferenciadas, oriundas do debate teórico e da prática de cada área do conhecimento, as quais, no entanto, muitas vezes se entrelaçam no cotidiano do bibliotecário em ciências da saúde.

Partindo dessa premissa, resolvemos investigar na literatura da área publicações que tratassem ao mesmo tempo das duas temáticas: Medicina Baseada em Evidências e Competência Informacional. Estabelecemos como primeiro objetivo de nosso trabalho conceituar Medicina Baseada em Evidências e Competência em Informação, e assinalar a confluência dessas temáticas na prática dos profissionais da área da saúde por meio de revisão de literatura.

Para tanto, realizamos uma busca em bases internacionais e periódicos nacionais na área de Biblioteconomia e C iência da Informação.

Foram pesquisadas as bases de dados internacionais: PubMed - indexed for Medline, Scopus, Library Literature \& Information Full Text, LISA: Library 
and Information Science Abstracts, Web of Science e LILACS: Literatura Latino-Americana e Caribe em Ciências da Saúde, utilizando os descritores: "evidencebased medicine" e "information literacy"

Não existindo bases de dados reconhecidas que indexam os principais periódicos nacionais na área de Biblioteconomia e C iência da Informação, realizamos uma busca nos sites dos seguintes periódicos: Ciência da Informação; Datagramazero; Em Q uestão: Revista da Faculdade de Biblioteconomia e Ciência da Informação UFRG S; Encontros Bibli: Revista Eletrônica de Biblioteconomia e Ciência da Informação; Perspectiva em Ciência da Informação; Revista ACB: Biblioteconomia em Santa Catarina; Revista Brasileira de Biblioteconomia e Documentação; Revista Digital de Biblioteconomia e Ciência da Informação e Transinformação em suas versões eletrônicas pesquisando o termo "medicina baseada em evidência". Essa busca não recuperou nenhum resultado e, por este motivo, não procedemos à pesquisa com 0 segundo termo "competência em informação".

0 critério utilizado para selecionar os periódicos para a pesquisa dos termos foi o uso da Lista $Q$ ualis da $C A P E S^{5}$ e incluídos aqueles com conceito $A$ e $B$, pois é uma referência amplamente utilizada nas Instituições de Ensino Superior, principalmente no que tange à pesquisa acadêmica.

\section{A Medicina Baseada em Evidências}

Dentre as muitas definições para Medicina Baseada em Evidências, destacamos a terminologia referida no Descritores em Ciências da Saúde - DECS, do Centro Latino-Americano e do Caribe de Informações em Ciências da Saúde da O MS/O PAS:

0 processo de procurar, avaliar e usar sistematicamente os achados de pesquisas contemporâneas como base para decisões clínicas. A medicina baseada em evidências faz perguntas, busca e avalia os dados relevantes, aproveitando as informações para a prática clínica diária, procurando seguir quatro passos: formular uma pergunta clínica clara a partir do problema de um paciente; procurar artigos clínicos relevan- tes na literatura; avaliar (criticamente) a validade e a utilidade das evidências; implementar osachados úteis na prática clínica. A expressão "medicina baseada em evidências" foi cunhada na McMaster Medical School (Canadá) nos anos 80 , para rotular a estratégia do saber clínico que o pessoal da faculdade desenvolvera durante mais de uma década (Centro..., Número do Registro: 33150, Identificador Único: D019317, 2007).

A chamada Medicina Baseada em Evidências (MBE) pode ser definida como o processo de sistematicamente descobrir, avaliar e usar achados de investigações como base para decisões clínicas. Tratase de uma prática que auxilia tanto profissionais da saúde quanto os pacientes a tomarem decisões com menor grau de incerteza, com base em evidências relevantes e reconhecidas.

No processo do desenvolvimento de decisão médica, tornaram-se imperiosos os aspectos científicos, com otimização dos benefícios, redução dos riscos e custos quando da aplicação dos recursos existentes (M ieli, 2003, p.142).

A MBE, segundo Sackett (2003, p.19), "é a integração das melhores evidências de pesquisa com a habilidade clínica e a preferência do paciente". Esses três fatores irão nortear a prática profissional daqueles que utilizam a informação como base para a clínica.

Para detectar a melhor evidência de pesquisa, a MBE trabalha com critérios de validade e relevância que orientam o profissional na forma correta de elaborar perguntas de aplicação clínica na assistência integral aos pacientes. Para Sackett (2003), os tópicos centrais dos quais surgem as perguntas clínicas são os achados clínicos, etiologia, manifestações clínicas da doença, diagnóstico diferencial, exames diagnósticos, prognóstico, tratamento, prevenção, experiência, significado e automelhora das habilidades do clínico em formular as perguntas. Sabe-se, no entanto, que:

toda a recomendação de uma conduta, por melhor que esteja embasada cientificamente (...) tem-se que levar em conta o caso específico de

\footnotetext{
5 O Q UALIS é uma classificação feita pela CAPES dos veículos utilizados pelos programas de pós-graduação para a divulgação da produção intelectual de seus docentes e alunos, cujo objetivo é atender às necessidades específicas da avaliação da pós-graduação realizada por essa agência. Disponível em <http://qualis.capes.gov.br/webqualis>. Acesso em: 11 de maio de 2007.
} 
cada paciente e o contexto em que se trabalha, e nisso a experiência de cada médico é muito importante (Atallah, 2001b, p.54).

Para Atallah (2003), a MBE veio também para, de maneira sistemática, aumentar os benefícios na utilização da ciência médica e o respeito aos direitos de pacientes. Um de seus princípios fundamentais é 0 compromisso do profissional de buscar a melhor informação científica para a solução de um problema de um determinado paciente. Diante disso, deve-se adequar a informação àquele paciente no seu contexto, com base na experiência do profissional, explicando detalhadamente 0 caso ao paciente, e, posteriormente, estando o médico bem informado cientificamente e 0 paciente esclarecido das melhores opções, to marem a decisão final em conjunto, responsabilizando-se ambos pelos resultados.

G reenhalgh (2005, p.19) propôs esta definição alternativa para a MBE:

Medicina baseada em evidências é o reforço das habilidades tradicionais de um clínico no diagnóstico, no tratamento, na prevenção e nas áreas relacionadas, por meio da formulação sistemática de questões relevantes e passíveis de serem respondidas, e o uso de estimativas matemáticas de probabilidade e risco.

A principal orientação para que o praticante da MBE se mantenha atualizado é a utilização de bancos de dados eletrônicos explícitos de evidência, periódicos em evidência e a consulta de sistemas computadorizados de apoio às decisões clínicas. 0 u ainda utilizar outras fontes de informação, responsabilizar-se pela análise das informações e buscar a evidência.

Segundo Atallah (2003, p.206-207), surgiu a necessidade de se mapear o conhecimento científico humano para cada problema médico.

Perguntas clínicas são então formuladas e 0 conhecimento cientifico existente é mapeado para se obter a melhor evidência científica para responder a cada questão. Esse processo é feito através de métodos científicos sofisticados denominados revisões sistemáticas e metanálises.

Algumas fontes de informações descrevem experiências analisa das e revistas por profissionais que estão constantemente testando as novas evidências, como é o caso da Biblioteca Cochrane, disponível online, que fornece revisões sistemáticas de ensaios de intervenções em assistência à saúde ${ }^{6}$.

Ao realizar buscas com o termo MBE nas principais bases de dados, o termo Prática Baseada em Evidências (PBE) é recuperado também, sendo um termo correlato.

A Prática Baseada em Evidências surgiu inicialmente no campo da Medicina, e, posteriormente, na Enfermagem. Nos últimos anos, nos eventos científicos e nas publicações nacionaise internacionais na área de enfermagem, o conceito de Prática Baseada em Evidências (PBE) tem recebido atenção de pesquisadores, educadores e enfermeiros assistenciais.

O s autores Stetler et al. (1998) apud Caliri e Marziale (2000) definem a Prática Baseada em evidências como uma abordagem para a enfermagem que utiliza os resultados de pesquisa, o consenso entre especialistas conhecidose a experiência clínica confirmada como bases para a prática clínica, ao invés de experiências isoladas e não sistemáticas, rituais e opiniões sem fundamentação. Para Madigan (1998) apud Caliri e Marziale (2000) 0 conceito de PBE surgiu no Canadá na área da medicina, como uma abordagem para resolução de problemas no ensino clínico e logo após foi incorporada pelo Sistema Nacional de Saúde do Reino Unido. N osEstados Unidos, o conceito foi utilizado poragências governamentais tanto para criar diretrizes e nortear políticas de assistência em diversas áreas, como para fornecer direcionamento aos profissionais so bre uma variedade de condições crônicas e agudas prevalentes no país.

\footnotetext{
6 A Biblioteca Cochrane, através da Biblioteca Virtual em Saúde (BVS), está disponível para todos os profissionais de saúde e de informação da América Latina e Caribe. 0 acesso na BVS a esta coleção de fontes de informação sobre evidências em saúde é produto de contratos de cooperação firmados entre a O rganização Pan-Americana da Saúde, por meio da BIREME, e as empresas Wiley Sons (US) e a Update Software (UK), com apoio da Colaboração Cochrane, do Centro Cochrane Ibero-Americano e do Centro Cochrane do Brasil. Disponível em:_<http://cochrane.bvsalud.org/portal/php/index.php?lang=pt>. Acesso em: 26 abr. 2007.
} 
A Prática Baseada em Evidências pode ainda ser definida como um méto do no qual os profissionais de saúde devem usar a melhor evidência possível para os cuidados em saúde, ou seja, a informação disponível ma is apropriada para tomada de decisões clínicas para pacientes individuais. A Prática Baseada em Evidências avalia, realça e se estrutura na perícia clínica, nos conhecimentos dos mecanismos da doença e na fisiologia. Ela envolve a complexa e consciente tomada de decisão baseada não somente na evidência disponível, mas também em características, em situações, e na preferência dos pacientes, reconhecendo que o cuidado de saúde é individualizado, sempre em mudanças e envolve incertezas e probabilidades. Finalmente a Prática Baseada em Evidências é a formalização do processo de cuidar que os melhores clínicos praticaram por gerações (M ckibbon, 1998).

\section{A Competência Informacional/Infor- mation Literacy}

No contexto internacional, a Association of College \& Research Libraries (ACRL), divisão da American Library Association, organizou um documento que define e fornece as aplicações de Information Literacy, o qual nos parece ser a base das definições adotadas na literatura nacional. Para essa instituição, Information Literacy fornece habilidades aos indivíduos que os tornam capazes de reconhecer quando uma informação é necessária, de forma que saibam localizar, avaliar e usar efetivamente a informação.

Ainda de acordo com a American Library Association (Association.., 2000) essas habilida des são cada vez mais exigidas, uma vez que proliferam mudanças nos recursos tecnológicos para a recuperação do grande volume de informação atualmente disponível. É comum a todas as disciplinas, a todos os ambientes de aprendizagem e a todos os níveis de instrução, a abundância de informação; entretanto, sem um conjunto de habilidades necessárias, o seu uso torna-se pouco eficaz. Para tanto é fornecido, nesse documento da $A C R L$, um conjunto de normas, indicadores de desempenho e resultados que podem ser aplicados na diversidade de áreas sugerida.

Belluzzo (2005a) cita que a noção de atitudes para o uso da informação é oriunda de movimentos paralelos em diferentes partes do mundo, a partir dos anos 80 .
Trata-se de um conjunto de atitudes referentes ao uso e domínio das tecnologias de acesso à informação: capacidades, conhecimentos e atitudes relacionadas com a identificação das necessidades de informa ção, conhecimentos das fontes de informação, elaboração de estratégias de busca e localização da informação, avaliação da informação encontrada, sua interpretação e síntese, reformulação e comunicação processos apoiados em uma perspectiva de solução de problemas e denominados como competência em informação (Belluzzo, 2005a, p. 39).

Estamos usando a expressão Competência Informacional como sinônimo de Information Literacy, a qual Dudziak (2003, p.28) define como "o processo contínuo de internalização de fundamentos conceituais, atitudinais e de habilidades necessário à compreensão e interação permanente com o universo informacional e sua dinâmica, de modo a proporcionar um aprendizado ao longo da vida".

Cavalcante $(2006$, p.48) comenta as discussões atuais relativas à competência informacional nas universidades, acentuando que, na educação superior, está relacionada principalmente com o uso das tecnologias, em diferentes suportes de informação, para favorecer o desenvolvimento das competências dos estudantes, o que beneficiará o crescimento profissional, a capacidade de realização de pesquisa, planejamento, gestão e avaliação no uso de fontes de informação.

Belluzzo (2006, p.83) destaca que "é importante também que as pessoas possam conhecer como 0 conhecimento está organizado, como buscar a informação, como utilizá-la de modo inteligente e como proceder ao processo de comunicação do conhecimento gerado", ou seja, a concepção do desenvolvimento da competência em informação está relacionada com a capacidade de criar significado, atitude fundamental no processo do aprendizado e geração do conhecimento.

0 paradoxo entre a remoção e as emergências de novas barreiras no acesso à informação são apresentadas por Belluzzo (2005b). Segundo a pesquisadora, com a evolução da Internet e sua utilização em larga escala, ocorre a remoção de inúmeras barreiras no acesso e uso da informação. Esse é, portanto, o passo inicial de todas as atividades de pesquisa e investigação, o qual permite às pessoas poderem acessar diretamente os documentos 
eletrônicos, independentemente de sua localização e sem intermediações. Existe, entretanto, um paradoxo nesse particular: inúmeras outras barreiras estão emergindo, em contrapartida, devido ao custo econômico-financeiro dessa tecnologia e também ao despreparo das pessoas em face da maior complexidade em relação aos processos de utilização adequada de fontes eletrônicas.

É preciso levar em consideração 0 aumento rápido e exponencial de informação que, muitas vezes, não tem a qualidade necessária, exigindo uma maior reflexão crítica sobre sua pertinência, relevância e confiabilidade.

A Ciência da Informação tem, entre seus objetivos, o desenvolvimento, gerenciamento e uso de fontes de informações confiáveis, e também a capacitação no uso das fontes de informações. Souto (2006) sugere que a Ciência da Informação exerce um papel importante na atualização dos profissionais da área da saúde, enquanto instrumento para a educação continuada, e evidencia os benefícios de se aplicar a Disseminação Seletiva da Informação na formação de profissionais da saúde.

Lopes $(2000, p .285)$ declara que a MBE se traduz pela prática da medicina em um contexto em que a experiência clínica é integrada com a capacidade de analisar criticamente e aplicar racionalmente a informação científica de forma a melhorar a qualidade da assistência médica. Dessa forma, alguém pode ser considerado possuidor das competências necessárias para a prática da MBE quando for capaz de:

identificar os problemas relevantes do paciente; converter os problemas em questões que conduzam às respostas necessárias; pesquisar eficientemente as fontes de informação; avaliar a qualidade da informação e a força da evidência, favorecendo ou negando o valor de uma determinada conduta; chegar a uma conclusão correta quanto ao significado da informação; aplicar as conclusões dessa avaliação na melhoria dos cuidados prestados aos pacientes.
Dessa forma, procurando identificar a confluência entre as duas temáticas: Medicina Baseada em Evidência e Competência em Informação, na prática dos profissionais da área de saúde, selecionamos do is trabalhos internacionais e o único trabalho nacional que encontramos considerados relevantes para esta análise, como segue:

A Divisão de Enfermagem da Universidade de $N$ ew York, para avançara prática baseada em evidência entre os clínicos, estudantes graduados e educadores, tomou a iniciativa de incluir componentes de Information Literacy em todo o núcleo do programa de mestrado.

De acordo com os autores J acobs, Rosenfeld e Harber (2003), o crescimento da Information Literacy é fundamental para a prática baseada em evidência e fornece para os profissionais cuidadores habilidades para serem "consumidores" de informação em um ambiente eletrônico. Isso inclui: compreensão da arquitetura da informação, do processo acadêmico, da habilidade de navegar entre uma variedade de ferramentas impressas e eletrônicas para acessar, buscar e avaliar eficazmente e criticamente os recursos apropriados; síntese da informação acumulada no conhecimento existente; comunicação, clara e eficaz de resultados de pesquisa; considerando 0 aspecto social e os interesses éticos relacionados à provisão, disseminação e compartilhamento da informação.

Honey, North e Gunn (2006), em pesquisa realizada em uma Universidade da Nova Zelândia, buscam identificar o uso da biblioteca pelos graduandos de enfermagem e as iniciativas tomadas pela biblioteca para divulgação de serviços e acessos, educação e promoção da competência em informação dos usuários. A Universidade da Nova Zelândia reconhece a Information Literacy como parte do conjunto de habilidades necessárias para os graduados de todas as áreas e concluem que a Information Literacy é um aspecto essencial para a educação dos profissionais de saúde, preparando-os para as mudanças no ambiente informacional global. $0 \mathrm{~s}$ autores acima consideram ainda a Information Literacy para os enfermeiros não apenas um simples conceito, mas um processo baseado nas definições de Information Literacy sugeridas pela American Library Association, incluindo: o reconhecimento das necessidades informacionais, 0 
conhecimento de como as informações são organizadas, e ainda, de como encontrar e usar a informação, o que significa compreender a Information Literacy como base para o aprendizado ao longo da vida.

No Brasil, destacamos uma pesquisa envolvendo um grupo de médicos residentes do Hospital Universitário Professor Edgar de Santos (HUPES), da Universidade Federal da Bahia, durante os meses de outubro a dezembro de 2004. 0 objetivo do trabalho foi definir o comportamento informacional do médico residente do HUPES, resultante das necessidades de informação científica surgidas durante sua prática clínica. Utilizando a técnica do survey - por meio da aplicação de um questionário-padrão - foram focalizados três aspectos-chave do fenômeno: (a) as necessidades informacionais; (b) o comportamento em relação às necessidades específicas de informação para a prática clínica - ou seja, a busca, o manejo e o acesso à informação -; (c) os serviços informacionais prestados por bibliotecas ou bibliotecários e os recursos eletrônicos implementados por profissionais da informação nesse contexto. 0 questionário foi enriquecido ainda com um bloco específico de perguntas que, utilizando a técnica do incidente crítico, levaram o participante da pesquisa a recordar e relatar em poucos passos um episódio recente que tivesse necessitado de informação (Martinez-Silveira; 0 ddone, 2005).

Consideramos importante 0 relato de experiências nacionais para 0 desenvolvimento de instrumentos de pesquisa e de metodologia.

\section{REFERÊN CIAS}

ASSO CIATIO N O F C O LLEG E \& RESEARC H LIBRARIES. Literacy Competency Standards for Higher Education. 2000. Disponível em: <http:// www.ala.o rg/ala/a crl/acrlstandards/ informationliteracycompetency.htm>. Acesso em: 9 maio 2007.

ATALLAH, A.N. Cochrane library gratuita. Revista Diagnóstico \& Tratamento, v.8, n.4, p.206-207, 2003.

ATALLAH, A.N. Princípios metodológicos para to mada de decisões médicas. Revista Diagnóstico \& Tratamento, v.6, n.1, p.47, 2001 .

ATALLAH, A.N. Tomadas de decisão em terapêutica. Revista Diagnóstico \& Tratamento, v.6, n.3, p.54, 2001b.

\section{CONSIDERAÇÕ ES FIN AIS}

Considera-se relevante a atuação do profissional da informação no contexto da prática de MBE devido ao seu papel de mediador entre usuários e estoques informacionais, corroborando a recuperação da informação.

É imprescindível saber como o conhecimento está organizado e como ele se apresenta, para se obter melhores resultados de busca, reduzir incertezas e ao mesmo tempo proporcionar aos profissionais de saúde maior suporte para tomada de decisão.

Percebeu-se, por meio deste trabalho, que 0 profissional da informação, como mediador, muito contribui para a melhor tomada de decisão na prática da MBE. Além disso, evidenciou-se a importância desse mesmo profissional na divulgação de serviços e acessos, na educação e na promoção da competência em informação dos pesquisadores.

As temáticas abordadas - Competência em Informação e Medicina Baseada em Evidências apresentam-se na teoria como um debate atual e na prática como um espaço de construções e descobertas.

Recomendamos pesquisas e estudos nacionais que envolvam as temática MBE e Competência em Informação com indicação de instrumentos validados para o uso dos profissionais da informação, que, assim como foi citado neste trabalho, é um profissional que busca 0 alinhamento de suas competências aos paradigmas em pauta.

BELLUZZO , R.C. Competências na era digital: desafios tangíveis para bibliotecários e educadores. ETD - Educação Temática Digital, v.6, n.2, p. 27-42, 2005a.

BELLUZZO , R.C. O uso de mapas conceituais e mentais como tecnologia de apoio à gestão da Informação e da comunicação: uma área interdisciplinar na competência em informação. Revista Brasileira de Biblioteconomia e Documentação: Nova Série, v. 2, n. 2, p.78-89, 2006

BELLUZZO , R.C. 0 uso de mapas conceituais para o desenvolvimento da competência em informação: um exercício de criatividade. In: PASSO S, R.; SANTO S, G .C. Competência em informação na sociedade da aprendizagem. Bauru: Kayrós, 2005b. 
BUEN O , S. B.; BLATTM AN N, U. Fontes de Informação on-line no contexto da área de Ciências da Saúde. Revista Digital de Biblioteconomia e Ciência da Informação, v.2, n.2, p. 1-17, 2005.

CALIRI, M.H.L.; MARZIALE, M.H.P. A prática de enfermagem baseada em evidências: conceitos e informações disponíveis online. Revista Latino Americana de Enfermagem, v.8, n.4, p.103-104, 2000. Disponível em: < http:// www.scielo.br/ pdf/rlae/v8n4/12391.pdf>. Acesso em: 11 maio 2007.

CAVALC ANTE, L.E. Políticas de formação para a competência informacional: o papel das universidades. Revista Brasileira de Biblioteconomia e Documentação: Nova Série, v.2, n.2, p.47-62, 2006.

CENTRO Latino-Americano e do Caribe de Informações em Ciências da Saúde. [Descritores em Ciências da Saúde]: Decs. 2007. Disponível em: < http:// www.bireme.br/php/decsws.php?>. Acesso em: 11 maio 2007.

DUDZIAK, E.A. Information literacy: princípios, filoso fia e prática. Ciência da Informação, v.32, n.1, p.23-35, 2003.

ELDIB, R.P.; ATALLAH, A.N. Cochrane library: como pesquisar? Revista Diagnóstico \& Tratamento, v.10, n.1, p.31-34, 2005.

FARIA, S. et al. Competências do profissional da informação: uma reflexão a partir da Classificação Brasileira de 0 cupações. Ciência da Informação, v.34, n.2, p.26-33, 2005.

G REEN HALG H, T. Como ler artigos científicos: fundamentos da Medicina Baseada em Evidências. Porto Alegre: Artmed, 2005.

HO NEY, M.; NO RTH, N.; G UN N, C. Improving library services for graduate nurse students in $\mathrm{N}$ ew Zealand. Health Information and Libraries Journal, v.23, p.102-109, 2006.

JACO BS, S. K.; RO SEN FELD, P.; HABER, J. Information literacy as the foundation for evidence-based practice in graduate nursing education: a curriculum-Integrated approach. Journal of Professional N ursing, v.19, n.5, p.320-328, 2003.
LE CO ADIC, Y.F. A Ciência da Informação. 2.ed. Brasília: Briquet de Lemos, 2004.

LO PES, A.A. Medicina Baseada em Evidências: a arte de aplicar o conhecimento científico na prática clínica. Revista da Associação Médica Brasileira, v.46, n.3, p.285-288, 2000.

MCKIBBO N, K.A. Evidence-based practice. Bulletin of the Medical Library Association, v.86, n.3, p.396-340, 1998.

MARTÍN EZ-SILVEIRA, M.S.; O DDO NE, N.E. Information-seeking behavior of medical residents in clinical practice. In: CO NG RESSO MUNDIAL DE INFO RMAÇÃO EM SAÚDE E BIBLIOTECAS, 9., 2005, Salvador. Anais eletrônicos. Disponível em: < http:// www.icml9.org/program/track10/ >. Acesso em: 14 fev. 2007.

MIELI, M.P.A. Senso comum, ciência e filoso fia. Revista Diagnóstico \& Tratamento, v.8, n.3, p.140-142, 2003.

O LIVEIRA, A.M.; et al. Mapeamento de competências em bibliotecas universitárias. Perspectivas em C iência da Informação, v.11, n.3, p.360-382, 2006.

PASSO S, R.; SANTO S, G.C. Competência em informação na sociedade da a prendizagem. Bauru: Kayrós, 2005.

PINTO, R.R. 0 profissional da informação em Ciências da Saúde: subsídios para o desenvolvimento de cursos de capacitação no Brasil. Dissertação (mestrado profissional) - Escola Paulista de Medicina. São Paulo: Escola Paulista de Medicina, 2005.

SACKETT, D.L. Medicina baseada em evidências: prática e ensino. 2.ed. Porto Alegre: Artmed, 2003.

SILVA, F.C.C. A atuação do bibliotecário médico e sua interação com os profissionais da saúde para busca e seleção de informação especializada. Revista Digital de Biblioteconomia e Ciência da Informação,v.3, n.1, p.131-151, 2005.

SO UTO , L.F. Disseminação seletiva da informação na área da Saúde: 0 caso do web site Amedeo. Revista Brasileira de Educação Médica, v.30, n.2, p.4-13, 2006. 
